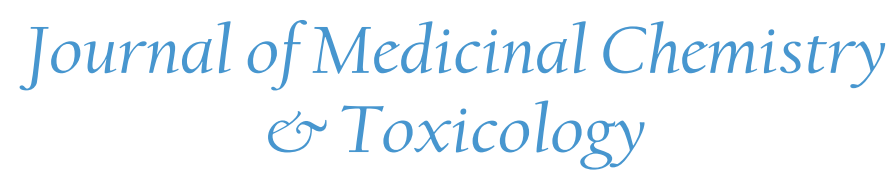

\title{
Medicinal Chemistry and Drug Designing
}

\section{Neelapu Nageswara Rao Reddy}

Department of Biochemistry \& Bioinformatics, Institute of Science, GITAM University, Visakhapatnam, A.P, India

Corresponding authors: Neelapu Nageswara Rao Reddy, Department of Biochemistry \& Bioinformatics, Institute of Science, GITAM University, Rushikonda, Visakhapatnam - 530045, A.P, India, Tel: +91-891-2840464, E-mail: nrneelapu@gmail.com

Citation: Neelapu, N.R.R. Medicinal Chemistry and Drug Designing. (2016) J Med Chem Tox 1(1): 15- 16.
Received Date: July 07, 2016

Accepted Date: July 14, 2016

Published Date: July 21, 2016

\section{Editorial}

Drug designing and development has its roots in age-old/traditional medicine and is changing with changes in medicinal chemistry. Age old medicines with good therapeutic potential were based on plant extracts. These medicines were effective, but drug targets were not known. In this regard more research should be extended to establish the target for the drug isolated from the natural product.

This may help the :

1) Pharmacists/pharmaceutical companies to generate information or have an idea on the drug targets and also their involvement in a particular disease

2) Indirectly help the pharmaceutical companies to identify a novel drug and thus to recover from the huge losses due the present business model.

3) Third world countries may find a solution to the diseases which are neglected till date.

Another important aspect in drug designing is to understand the principal component in the natural product that is actually having the therapeutic benefit.. This would benefit the medicinal chemists to develop an analogue with biological activity similar to that of natural product. Medicinal chemistry plays an important role in developing a drug with therapeutic benefit. Medicinal chemistry is traditionally modifying a chemical molecule (natural product) which is of therapeutic value to intervene a disease. This includes identifying a chemical molecule of potential, altering its chemical structure, synthesizing the organic molecule in the laboratory, and testing of its properties and biological activities. Medicinal chemistry has driven the market of the pharmaceutical industry and is continuing to do so.

Medicinal chemistry has witnessed changes in the process of drug designing and development. The primary aim of the present day drug designer is to design a drug for a known target and develop a molecule by completely understanding the active principle with therapeutic potential. Present day drug discovery and development follows a very systematic and complex process to generate Food and Drug Administration (FDA) approved drugs. The drug discovery process starts with identification and validation of drug targets for a particular disease ${ }^{[1-5]}$. Medicinal chemists design a new molecule to the established target using Computer Assisted Drug Designing (CADD).

CADD helps medicinal chemist in screening of small molecule library to identify and establish hit by a process known as virtual screening. Computational chemistry helps medicinal chemist in hit to lead optimization by modifying the hit and retaining the pharmacological properties of hit. The hit to lead optimization is done by employing molecular dynamics, molecular simulations, Structure Activity Relationship (SAR) and Quantitative Structure Activity Relationship (QSAR). . Further lead optimization is done by employing molecular docking and by performing Absorption, Distribution, Metabolism, and Excretion (ADME) profile.

In summary, identifying biological targets for age old/traditional medicines, identifying the core component with therapeutic value in the natural product and implementation of methodologies and advanced computing by medicinal chemists would drive or revolutionize drug designing and medicinal chemistry.

Copyrights: (C) 2016 Neelapu, N.R.R. This is an Open access article distributed under the terms of Creative Commons Attribution 


\section{References}

1. Dutta, A., Singh, S.K., Ghosh, P., et al. In silico identification of potential therapeutic targets in the human pathogen Helicobacter pylori. (2006) In Silico Biol 6(1-2): 43-47

2. Neelapu, N.R.R., Pavani, T. Identification of novel drug targets in HpB38, HpP12, HpG27, Hpshi470, HpSJM180 strains of Helicobacter pylori: an in silico approach for therapeutic intervention. (2013) Curr Drug Targets 14(5): 601-611

3. Neelapu, N.R.R., Srimath-Tirumula-Peddinti, R.C., Nammi, D., et al. New strategies and paradigm for drug target discovery: A focus on infectious diseases tuberculosis, gastritis, malaria, trypanosomiasis and leishmaniasis. (2013) Infect Disord Drug Targets 13(5): $352-364$

4. Neelapu, N.R.R., Naresh, M.V.R., Srinivas, A. Identification of potential drug targets for Helicobacter pylori strain HPAG1 by in silico genome analysis. (2015) Infect Disord Drug Targets 15(2): 106-117

5. Nammi, D., Srimath-Tirumala-Peddinti, R.C., Neelapu, N.R.R. Identification of drug targets in Helicobacter pylori by in silico analysis: possible therapeutic implications for gastric cancer. (2016) Curr Cancer Drug Targets 16(1): 79-98

Ommega Online Publisher

Journal of Medicinal Chemistry \& Toxicology

Short Title : J Med Chem Toxicol
ISSN No: 2575-808X

E-mail: medchemtoxic@ommegaonline.org

website: www.ommegaonline.org 